opportunities for using emerging technologies in interventions and training. Our next project is a VR tour of our inpatient unit and grounds filmed using a 360 degree camera for patients to watch at home to try and alleviate some of the worries and anxieties they may have about staying with us or accessing our services.

Conclusion The use of VR in healthcare has endless possibilities. Working with our local education partners is an opportunity to explore this for our patients. Positive outcomes have provided the impetus to try new approaches that will have real impact on patient wellbeing and symptom management.

\section{P-138 EXPANDING THE REACH OF VIRTUAL REALITY FOR PALLIATIVE PATIENTS}

Sheila Popert, Kreeti Magar. Prospect Hospice, Swindon, UK

\subsection{6/spcare-2021-Hospice. 155}

Background The use of virtual reality (VR) for symptom management in palliative care has grown over the last five years; most of the studies have been of its use in hospice (Popert $\&$ Riat, 2017) and hospital inpatient (Nwosu, Mills, Roughneen, 2021; Niki, Okamoto, Maeda, et al., 2019) and day therapy units (DTU). The advent of the COVID-19 pandemic resulted in the temporary closure of our DTU, so we therefore also offered patients the opportunity to use the headsets in their own homes.

Aim This study explores the impact of using VR on reported pain, anxiety and mood in palliative patients and the feasibility of using it in the home.

Method Patients are instructed how to use VR headsets on the inpatient unit or in their own home. Participants complete questionnaires on its influence on pain and anxiety scores and their experience of use. Volunteers were trained to teach patients how to use the headsets.

Results 30 sets of data have been collected from the inpatient unit and 10 from patients' homes, results for both groups are similar. All patients reported ease of use, a positive experience, and no adverse effects. 98\% of those who reported pain before using VR reported a reduction and 93\% of those who reported anxiety before using VR reported a reduction. For the majority of participants in this study using VR induced emotion with positive valence - happiness, excitement. The ability of VR to induce emotions with positive valence is deemed to influence its analgesic effect (Triberti, Repetto, Riva, 2014).

Conclusion The majority of palliative patients are managed in the community setting, and enabling the use of VR in their own homes means more patients can experience the benefits. There are multiple small studies on the impact of VR on symptoms in palliative patients but randomised controlled trials are needed (Austin, Lovell, Siddall, 2019). It is an intervention that puts a smile on patients' faces.

\section{P-139 EMERGENCY SYMPTOM CONTROL NEAR THE END-OF- LIFE: A CLINICAL AUDIT EXAMINING AMBULANCE CLINICIAN CARE}

Karina Catley, Georgina Murphy-Jones, Diane Laverty. London Ambulance Service NHS Trust, London, UK

10.1136/spcare-2021-Hospice.156
Background The London Ambulance Service NHS Trust (LAS) performs a vital role in the assessment and management of symptoms in end-of-life care (EoLC) patients during times of crisis. A previous clinical audit identified symptom control as an area requiring improvement. Following funding of a Macmillan EoLC team and release of national guidance, a re-audit was conducted to assess symptom control by ambulance clinicians. Aim Review ambulance clinicians' assessment and management of symptoms for patients nearing their end-of-life.

Method A retrospective clinical audit was conducted using data from 01/11/2019- 30/11/2019. From a total of 363 cases, 58 were excluded due to the patient having no identified EoLC needs. A resulting sample of 305 patient report forms were clinically reviewed.

Results Several elements of practice were encouraging but results highlighted a number of areas for improvement:

- Pain assessment: $84 \%$ of patients had an appropriate pain assessment documented.

- Pain management: 65\% of patients presenting with pain/ discomfort had analgesia administered by the attending ambulance clinicians.

- Pharmacological symptom control: 61\% of patients received an appropriate pharmacological intervention when necessary. This included providing LAS issued medication for nausea/ vomiting or anticipatory medications.

- Non-pharmacological symptom control: non-pharmacological interventions (e.g. re-positioning for breathlessness) were documented for $18 \%$ of eligible patients.

Conclusion End-of-life care is a novel area of pre-hospital care and whilst service improvement progresses (Murphy-Jones, Laverty, Stonehouse, 2021) it will take time to embed guidance and education into practice. Inherent actions in pre-hospital practice, such as non-pharmacological interventions may be undertaken but not documented and thus not captured by the audit process. It is clear however that EoLC symptom control requires improvement. The Macmillan EoLC team have shared findings with staff to highlight areas for improvement, created new guidance and enhanced education, covering both pharmacological and non-pharmacological symptom control. A continuous EoLC clinical audit within the Trust has been introduced to monitor and improve compliance.

\section{P-140 SINGLE NURSE ADMINISTRATION OF DRUGS IN A HOSPICE IPU}

Laura Speight, Clare Dixon. St Gemma's Hospice, Leeds, UK

10.1136/spcare-2021-Hospice. 157

Background St Gemma's Hospice recognises the national shortage of registered nurse (RN) recruitment with increasing dependency of patients (The Kings Fund. The NHS workforce: our position, 2021). As part of our ongoing development and cost effective use of resources, the nursing team are keen to use the existing $\mathrm{RN}$ resource as efficiently as possible. By moving to a workplace where it is usual practice for a single nurse to check and administer drugs (SNAD) would enable greater safety, more timely symptom management and more effective use of the workforce (Cross, Bennett, Ockerby C, 2017; Armitage, 2008).

Aim Inpatient unit (IPU) moves to single nurse administration of most drugs by June 2021 . 